\title{
Análisis del contenido intestinal en momias
}

Claudio Paredes ${ }^{1}$ y Eugenio Aspillaga ${ }^{1}$

\section{Introducción}

De todos los elementos biológicos que nos puede entregar el estudio de un resto humano momificado, como patológicos, biométricos y otros, el más apropiado para entregar la mayor cantidad de información respecto del ambiente es el análisis del contenido intestinal, pues nos permite, hasta cierto punto, determinar la composición de la dieta, e inferir algunos factores ambientales. Es decir, es posible reconstruir, en parte, la ecología de un grupo humano, pero el análisis del contenido intestinal debe delimitarse a las zonas anatómicas del sistema digestivo, pues nos encontramos con el siguiente objeto de estudio: elementos de la naturaleza masticados y prácticamente desintegrados, según la zona del tracto digestivo en que se encuentran. Para su estudio se deberán considerar los elementos de análisis que detallamos a continuación:

\section{Aspectos metodológicos}

Deberá seguirse un estricto protocolo de análisis, en primer lugar, de los elementos externos de la momia para luego proceder a la disección de ésta.

1. Análisis externo. Debe estudiarse el cuerpo siguiendo un determinado orden, extremidades inferiores, tronco, extremidades superiores y cabeza.

a) Posición. Se considera la posición relativa de los elementos anatómicos entre sí, lo que determinará la forma y posición externa total del cuerpo, esto es importante porque el individuo al ser depositado sus vísceras pueden desplazarse por acción de la gravedad, además, después de disectado el cuerpo será prácticamente irreconstituible; por lo cual hemos ideado un método para dejar registrada la forma externa de una momia y las relaciones de los

1 Departamento de Ciencias Sociológicas y Antropológicas de la Universidad de Chile. Larraín 9925, La Reina, Santiago, CHILE. órganos internos, trabajo que es presentado también en este Simposio (Aspillaga et al. 1983 Ms).

b) Estado de conservación. Se deberá observar y registrar el estado de conservación de los elementos anatómicos, tomando en cuenta los siguientes puntos: a) El material correspondiente al tejido blando es inexistente o escaso, pudiendo estar destruidas las vísceras; b) Existencia de rastros de descomposición, por la posición del cadáver o por condiciones ambientales como persistencia de humedad; c) Contaminación. Existencia de erosiones y perforaciones si el cuerpo ha estado expuesto al aire libre que se hayan producido en la excavación y extracción del cuerpo, ya sea al ser transportado, o bien intencionales para otros estudios. También cuando el cuerpo ha estado guardado en una bodega o expuesto en exhibición. Otro agente de contaminación son los agregados químicos que se colocan al cuerpo para prevenir infestaciones de insectos y otros o cuando se encuentran en bodegas y/o en exhibición. Se ha descrito un excelente método de autopsia de una momia, el cual ha sido ampliamente aplicado (Allison 1982).

2. Disección. Observados y descritos los caracteres del análisis externo, se procede a la disección. En ocasiones es necesario retirar las extremidades inferiores y superiores, por cuanto la momia puede encontrarse en posición flectada y los brazos descansando sobre el cuerpo. También es posible separar la cabeza para estudios craneométricos.

a) Quedando el tronco sin extremidades y sin cabeza: Se procederá a hacer un corte, denominado en delantal en disección anatómica. Este recorre el borde inferior del tórax siguiendo la línea de las costillas, continúa por los lados del cuerpo hasta llegar a la espina ilíaca anterosuperior y se dirige hacia delante hasta el borde superior de la hoja del pubis. Se levanta la pared abdominal dejando todo el abdomen expuesto. También se separa la caja torácica, seccionando a nivel de los cartílagos 
esternales, siguiendo el corte sobre las clavículas y desarticulando las costillas con la columna vertebral. Luego se levanta la caja torácica, dejando expuesto el tórax; de esta manera queda expuesto todo el tracto digestivo.

b) Se procede a su inspección, considerando coloración, textura y forma, ver si hay patologías y desplazamientos; a continuación, se secciona longitudinalmente el tracto digestivo dejando visible su interior, donde se observan las características del contenido fijándose en los sectores donde de encuentra acumulado el contenido; se indica la cantidad en volumen del contenido de cada sector.

c) Se extraen muestras del contenido con instrumentos tales como espátulas, pinzas y cuchillos finos. La cantidad de las muestras deberá ser equivalente con el fin de poder hacer recuentos de densidades relativas de cada elemento, indicando además cantidades precisas en volumen y peso. Los sectores a extraer muestras pueden ser: esófago cuando haya habido regurgitación de alimentos, estómago, intestino delgado, duodeno, yeyuno o íleon y, por último, intestino grueso, como ser colon ascendente, colon transverso, colon descendente o colon ileo-pélvico.

3. Muestras. Cada muestra extraída se describe microscópicamente, considerando relación peso y volumen, coloración general, textura, así como si hay elementos reconocibles y las características de cada uno. Luego se describen, observándolos a la lupa estereoscópica o microscopio en lupa, indicando las características, detallando los elementos reconocibles y señalando en algunos casos cuándo es necesario hacer incremento de las intensidades de aumento. Usualmente se aplica $20 \mathrm{X}$, luego se aumenta a 40 X y $100 \mathrm{X}$, después a $500 \mathrm{X}$ y $1000 \mathrm{X}$ cuando los elementos más pequeños como huevos y quistes de parásitos quieren reconocerse. Posteriormente, se hidrata parte de la muestra en una cantidad determinada en agua destilada (o mejor en una solución hipotónica), dejándola un tiempo prudente, el que siempre es variable por la humedad relativa de los tejidos, hasta que éstos se hagan elásticos a la tracción. Primero se describen las mismas características de la muestra seca a ojo desnudo y luego, colocando la muestra hidratada bajo lupa estereoscópica o en microscopio en lupa (incrementando la intensidad del aumento en ocasiones) para reconocer algunos elementos.
4. Tratamientos especiales. El método descriptivo anterior no altera la muestra, pero es posible aplicar a parte de ésta tratamientos físicos y químicos especiales con el fin de identificar elementos de la dieta y extraer resultados más específicos que la simple descripción del contenido intestinal.

Para el reconocimiento del material vegetal una técnica de preparación de los tejidos, que hemos modificado, se especifica en el Anexo 1. Los tejidos epidérmicos vegetales se reconocen fácilmente, pero para un reconocimiento efectivo es absolutamente necesario disponer de observaciones previas de especímenes conocidos y confeccionar su propio catálogo.

El examen parasitológico simple es otro método adecuado para ser aplicado a estos estudios, ya que no es posible aplicar el método de recolección anal porque los huevos y quistes han perdido su potencialidad. El método se describe en el Anexo 2. Se observa primero con lupa (20 X) para determinar la ubicación de la muestra y se incrementa la intensidad; con más de 100 aumentos es posible distinguir algunos huevos y quistes de parásitos o a estos mismos.

Otro tratamiento especial factible de realizar a muestras de contenido intestinal de una momia es la concentración de hidrogeniones, descrita en el Anexo 3. Además para fines de comparación de la dieta se determina la fracción animal y la fracción vegetal, método que hemos llamado disgregación con $\mathrm{HNO}_{3}$ y que describimos en el Anexo 4; la comparación se realiza a través de los volúmenes de la parte sólida.

\section{Aplicación del método}

Este método es aplicable a un determinado tipo de momias, sean naturales por desecación o por congelación o artificiales sin evisceración, tanto antiguas como modernas (pre y postcolombinas). La condición es que presenten sus vísceras en un adecuado estado de conservación y que hayan sido debidamente excavadas y guardadas.

Se analizaron 12 momias en excelente estado de conservación, provenientes del sitio Caserones, de las cuales se extrajeron varias muestras del contenido de distintos sectores del tracto digestivo. Estas momias forman parte, además, de otros estudios del Laboratorio de Antropología Física de la Universidad de Chile. 
Al disectar estas momias y exponer el interior del tórax y del abdomen se presentan los órganos internos dispuestos anatómicamente con pequeñas alteraciones en su posición relativa. Según la postura que tenía el individuo en la tumba, hay un cierto desplazamiento de los órganos y en algunos sectores que no tienen contenido los tejidos se encuentran adheridos. La única diferencia con el vivo es que en las momias los órganos se encuentran igual pero desecados y ligeramente aplastados contra la zona donde descansaba el cuerpo. Otra diferencia es en la coloración de los órganos. Estos presentan un color pardo, en general café rojizo brillante, correspondiente a las grasas oxidadas. Pero esta coloración no es homogénea, por lo que es fácilmente identificable el tracto digestivo y además por la persistencia de la posición anatómica.

Los elementos encontrados e identificados se pueden resumir en el Cuadro 1, pero el detalle específico se resume de la siguiente manera:

Estómago. Se encontraron semillas y fragmentos de éstas del género Prosopis, restos de hojas, fibras vegetales, cristales grasos, pelos de roedor pequeño, arena, tallos de gramíneas y vegetales superiores, carbón, restos de insectos. Como contaminación, larvas y huevos de derméstidos; $\mathrm{pH} 4$ a 5; al tratar la disgregación con $\mathrm{HNO}_{3}$ se estimó en 3/4 la parte vegetal y en 1/4 la fracción animal; es decir, una relación de 3:1.

Intestino delgado. Arena, fibras y tejidos vegetales, cristales grasos y de líquidos corporales, carbón, restos de insectos, fibras animales. Contaminación de hongos y derméstidos.

Colon ascendente. Arena, fibras y tejidos vegetales, cristales grasos y de líquidos corporales, esferas grasas, huevos de parásitos sin identificar, carbón. Contaminación de hongos y derméstidos, pH 8.

Colon transverso. Arena, fibras y tejidos vegetales. Cristales de grasas, líquidos corporales, oxalato, fosfato triple. Quistes de Entamoeba coli. Contaminación de hongos y derméstidos.

Colon descendente y recto. Arena, fibras y tejidos vegetales, cristales de grasas, fosfato triple, oxalato y líquidos corporales. Quistes de Entamoeba coli. $\mathrm{Al}$ aplicar disgregación con $\mathrm{HNO}_{3}$ la fracción sólida era casi completamente vegetal.
Si bien es cierto que en todo el tracto digestivo los elementos vegetales y animales son los mismos, se observa claramente el hecho de que van disminuyendo los tamaños relativos de la fracción vegetal, permaneciendo reconocibles los tipos de tejidos y fibras de la sección a la que ellos pertenecen. También la raíz, tallo u hoja, identificables por sus distintas reacciones a los pigmentos (p.e., celulosa) responde a la zafranina. Además, son identificables los distintos tipos de tejidos de origen animal; así con gran aumento se reconoce el músculo estriado. La fracción animal también se desintegra paulatinamente al avanzar por el tracto digestivo hasta desaparecer los fragmentos, distinto a lo que ocurre con la fracción vegetal.

Sería sumamente útil presentar a futuro los resultados de los elementos reconocidos en un cuadro comparativo, seleccionando por una entrada el tipo de individuos (niños, juveniles, distintos grupos de adultos) por sexos; y por la otra entrada los distintos tipos de fragmentos.

\section{Comentarios}

La aplicación del método a un sitio específico como Caserones nos entrega una reconstrucción tentativa y cualitativa del ambiente de este grupo humano. Principalmente éstos disponían de elementos vegetales como raíces, tallos y hojas de plantas superiores, algunas gramíneas y Prosopis. Ello permite deducir que su ambiente es el típico oasis de desierto. La presencia principalmente de Prosopis en biocenosis con gramíneas y plantas superiores y asimismo la escasa cantidad de plantas acuáticas confirman lo anterior. Por otra parte, la muy baja frecuencia de elementos ajenos al ambiente indicado es otro hecho importante. Se presenta una baja frecuencia de restos animales, siendo escasos los de roedor y pescado y otros restos de origen animal, lo que sugiere que el grupo humano tenía una baja actividad de acceso o intercambio con ambientes distintos al oasis de desierto. Por lo tanto, la función ecológica (nicho) es una utilización mezclada de recursos, consumo de primer y segundo orden, es decir, un sistema recolector y cazador de poca envergadura sobrepuesto al uso del sistema principal, un oasis de desierto.

El método presentado supra y aplicado a un ejemplo particular es absolutamente factible si se repitiese en momias de otros sitios arqueológicos, entonces será posible comparar los resultados obtenidos de los distintos sitios. Las comparaciones pueden 


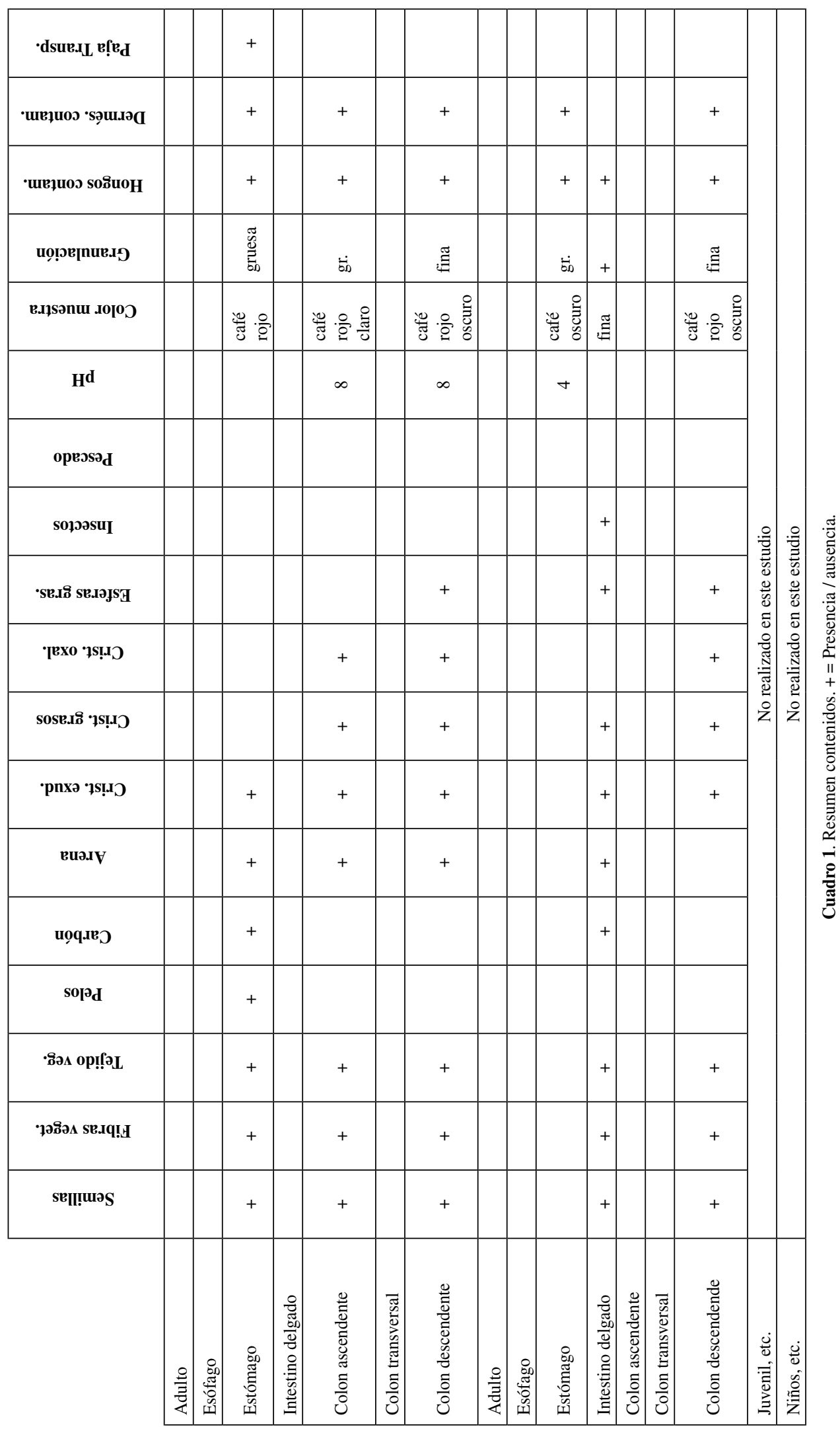


efectuarse de lo extraído del material biológico con lo obtenido del registro arqueológico; entonces se reconstituirán en su mejor forma las culturas pasadas (objeto de la arqueología): uniendo el objeto protagonista, o sea hombre, con lo que efectúa: la cultura.

Del análisis del contenido estomacal e intestinal es posible obtener una cantidad apreciable de resultados inferidos o directos que pueden ser útiles como datos para investigaciones posteriores:

- Composición de la dieta.

- Calidad de la dieta, elementos de higiene y valor nutricional de la dieta.

- Parasitosis.

- Epidemiología de las enfermedades parasitológicas.

- Recursos faunísticos y florísticos del ambiente (ecosistemas accesibles).

- Relaciones entre los elementos del ambiente.

El método planteado, si bien es sencillo de aplicar, requiere que algunos pasos sean realizados por una persona relativamente experta; como ejemplo, uno de los problemas más difíciles de enfrentar es la identificación de huevos y quistes de parásitos, por lo cual el determinador deberá tener una cierta experiencia previa. Existen otros análisis, no aplicados aquí, pero que son posibles de efectuar como el análisis palinológico, la electroforesis, el análisis ciclotrónico, la microscopía electrónica simple, la microscopía electrónica de barrido (scanning) y la tomografía computarizada (scanner).

\section{Conclusiones}

El objetivo de este trabajo fue mostrar el camino que puede seguirse en el análisis de los restos humanos, además de estudios de este tipo, los cuales aplicando la tecnología adecuada aportarán más resultados y datos a nuestras disciplinas. Como en el ejemplo presentado del sitio Caserones, mayores estudios sobre esa muestra y en otros restos de la zona contrastados con las referencias arqueológicas podrán ser motivo de otras investigaciones.

\section{Anexo 1}

\section{Técnica de preparación del material vegetal}

1. Se toma una muestra del material a analizar, se incluye en hidróxido de sodio al 7\%, durante algunas horas.
2. Se lava la muestra.

3. Se incluye en solución de Schultze, ácido nítrico y clorato de potasio al 7\%, en proporción 1 a 1.

4. Se incluye en solución saturada de zafranina.

5. Se lava suavemente, con el fin de eliminar la zafranina excedente.

6. Se coloca sobre un portaobjeto, se cubre con cubreobjeto y se observa al microscopio.

\section{Anexo 2 \\ Técnica para examen parasitológico}

1. Se toma una muestra del material a analizar, se hidrata, a esta emulsión se le agrega el mismo volumen de formol sal.

2. Se tamiza a través de una fina malla de cobre, la emulsión se vierte en un tubo.

3. En un tubo de centrífuga se coloca la emulsión filtrada, hasta llenar el tubo 3/4 del total, se agrega éter, hasta llenar a 1 centímetro del borde superior del tubo, se agita enérgicamente y se destapa lentamente.

4. Se centrifuga 4 a 5 minutos a $2000 \mathrm{rpm}$, se vacía el contenido con golpe seco y se deja boca abajo a fin de eliminar la mayor cantidad posible de líquido, se agita el contenido con pipeta Pasteur despuntada y se aspira una pequeña cantidad.

5. Se deposita una gota de lugol en un portaobjeto y se agrega una gota de sedimento, se esparce con el vértice del cubreobjeto, se cubre con éste, evitando absolutamente las burbujas y se observa al microscopio.

\section{Anexo 3 \\ Técnica para determinar el $\mathbf{p H}$}

1. Se toma una muestra del material a analizar, se hidrata con agua destilada o con suero fisiológico de $\mathrm{pH} 7$.

2. Se coloca en contacto de la emulsión fecal un trozo de papel $\mathrm{pH}$.

3. Se compara con la tabla de colores y correspondientes $\mathrm{pH}$, de la caja donde viene el papel. 


\section{Anexo 4 \\ Técnica de disgregación con $\mathrm{HNO}_{3}$}

1. Se toma una muestra del material a analizar, se hidrata.

2. Se le agrega igual volumen de ácido nítrico concentrado, $\mathrm{pH} 2$, luego se mezcla con varilla de vidrio hasta transcurrir 1 hora.
3. Se comparan los volúmenes de la parte sólida, extrayendo el agua y el ácido nítrico. La fracción vegetal permanece intacta y la fracción animal se disuelve.

\section{REFERENCIAS CITADAS}

ALLISON, M. J. y E. GERSZTEN, 1982. Paleopathology in South American mummies. College of Virginia, Virginia Commonwealth University, Virginia.

ASPILLAGA, E., A. PAREDES y M. GONZALEZ, 1983Ms. Método de registro gráfico de la forma externa de una momia. Ponencia presentada al Simposio de Arqueología Atacameña, San Pedro de Atacama.

ERDOS, J. y M. SPIERA, 1944. Métodos clinicoquímicos de laboratorio. Editorial Atlante, México D. F.
FOUANT, M., M. ALLISON, G. FOCACCI y E. GERSZTEN, 1982. Parásitos intestinales entre los indígenas precolombinos. Chungara.

KRUPP, M., N. SWEET, E. JAWETS y E. BIBLIERI, 1973. Prontuario médico. Manual Moderno S.A., México D. F.

ROUVIERE, H., 1959. Compendio de anatomía y disección. Salvat S.A., Barcelona.

TABLAS CIENTIFICAS, 1965. Documenta Geigy, Basilea. 